\title{
Whole-blood PUFA and associations with markers of nutritional and health status in acutely malnourished children in Cambodia
}

\author{
Sanne Sigh ${ }^{1,2, *}$ (1), Lotte Lauritzen ${ }^{1}$ (1), Frank T Wieringa ${ }^{3}$, Arnaud Laillou ${ }^{4}$, \\ Chhoun Chamnan ${ }^{2}$, Nheb Angkeabos ${ }^{5}$, Duch Moniboth ${ }^{5}$, Jacques Berger ${ }^{3}$, Ken D Stark ${ }^{6}$ \\ and Nanna Roos ${ }^{1}$ \\ 'Department of Nutrition, Exercise, and Sports, Faculty of Science, University of Copenhagen, Frederiksberg, \\ Denmark: ${ }^{2}$ Department of Fisheries Post-Harvest Technologies and Quality Control, Fisheries Administration, \# 186 \\ Preah Norodom Boulevard, 12101 Phnom Penh, Cambodia: ${ }^{3}$ Institut de recherce pour le dévelopment (IRD)/ \\ Université de Montpellier/SupAgro, Montpellier, France: ${ }^{4}$ Department of Child Survival and Development, UNICEF \\ Cambodia, Phnom Penh, Cambodia: ${ }^{5}$ National Pediatric Hospital, Phnom Penh, Cambodia: ${ }^{6}$ Department of \\ Kinesiology, University of Waterloo, Waterloo, Ontario, Canada
}

Submitted 25 February 2019: Final revision received 27 July 2019: Accepted 20 August 2019: First published online 24 January 2020

\begin{abstract}
Objective: To measure fatty acid composition, particularly whole-blood PUFA content, in acutely malnourished children and identify associations with markers of nutritional and health status.

Design: PUFA were assessed in dried blood spots obtained from a cross-sectional study. Nutritional and health status were assessed by anthropometry, haemoglobinopathies, inflammation and blood counts.

Setting: Cambodia.

Participants: The study was conducted with 174 children aged 0.5-18 years with acute malnutrition.

Results: Among total fatty acids (FA), the relative percentage of total PUFA was $20 \%$ FA, with $14 \%$ of the children having very low PUFA (mead acid (MA): arachidonic acid (AA) $>0.02, n-6$ docosapentaenoic acid:DHA $>0.2$ and total $n-6: n-3$ PUFA $>10 \cdot 5)$. Wasting was not associated with any PUFA. Stunting and low height were consistently positively associated with total PUFA and positively with n-6 PUFA. Height was positively associated with $n$-3 long-chain PUFA (LCPUFA). The presence of haemoglobinopathies or inflammation was positively associated with MA:AA, but not total PUFA. Elevated blood platelet counts were positively correlated with linoleic acid and appeared to be influenced by anaemia $(P=0.010)$ and inflammation $(P=0.002)$. Monocyte counts were high during inflammation $(P=0.052)$ and correlated positively with $n-6$ LCPUFA and $n-3$ LCPUFA.

Conclusions: Children with acute malnutrition or stunting had low PUFA, while elevated platelets and monocytes were associated with high PUFA. In acutely malnourished children, inflammation could lead to elevated blood cell counts resulting in increased whole-blood PUFA which does not reflect dietary intake or nutritional status.
\end{abstract}

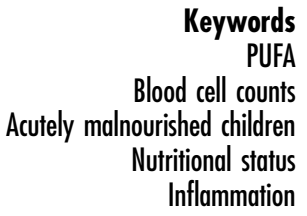

PUFA

(l)
Acute malnutrition is a prevalent public health problem in many low- and middle-income countries. It contributes significantly to childhood morbidity and mortality and affects more than 70 million children annually ${ }^{(1,2)}$. Acute malnutrition is partly caused by insufficient intakes of energy and nutrients for maintaining essential body functions. The food consumption in low- and middle-income countries is often a starch-based diet such as rice, legumes, fruits and vegetables that is low in PUFA ${ }^{(3,4)}$.

Dietary linoleic acid (18:2n-6; LA) and $\alpha$-linolenic acid (18:3n-3; ALA) are essential fatty acids and can be metabolized to arachidonic acid (20:4n-6; AA) and DHA (22: 6n-3), respectively. These long-chain PUFA (LCPUFA) are critical for healthy growth, immune function, brain 
development and the skin-water barrier ${ }^{(5-7)}$. Acutely malnourished children may be particularly vulnerable to essential fatty acid deficiency owing to low food consumption, disturbed lipid metabolism and absorption, and/or diarrhoea caused by intestinal infections ${ }^{(8-10)}$.

In children with moderate acute malnutrition (MAM) and/or severe acute malnutrition (SAM), PUFA status has been shown to be altered in different blood pools such as sterols ${ }^{(11)}$, triacylglycerols ${ }^{(12)}$, plasma ${ }^{(13,14)}$, erythrocytes $^{(15)}$ and whole blood ${ }^{(16-18)}$. Whole-blood samples have logistical advantages for field research collection due to less sample processing immediately post collection, as well as the ability to collect dried blood spots. However, whole blood includes plasma and various types of blood cells that can differentially impact the overall fatty acid composition ${ }^{(19)}$. Acutely malnourished children living under poor conditions are vulnerable to infections, inflammation, anaemia, and other nutritional and health challenges ${ }^{(10,20-24)}$ that could affect blood cell counts, such as increased monocytes with infection ${ }^{(25,26)}$. Changes in cell counts can affect the overall fatty acid composition of whole blood, which could alter interpretations of whole-blood PUFA status. Another health challenge that is prevalent in South-East Asia is genetic Hb disorders (haemoglobinopathies), which may contribute to changes in whole-blood PUFA composition due to increased risk of anaemia and altered erythrocytes caused by these disorders $^{(27,28)}$.

The purpose of the present study was to investigate the fatty acid composition, particularly the PUFA content, of whole blood and potential associations with markers of nutritional and health status in acutely malnourished Cambodian children.

\section{Methods}

\section{Study design and setting}

The present cross-sectional study uses baseline data from an 8-week interventional trial evaluating the effectiveness of a locally produced, fish-based, ready-to-use therapeutic food compared with a standard ready-to-use therapeutic food during outpatient treatment of acute malnutrition in Cambodian children (called the 'SAM trial') ${ }^{(29,30)}$. The SAM trial was conducted at the National Pediatric Hospital in Phnom Penh, Cambodia. The sample size in the trial was based on the superiority of one of two ready-to-use therapeutic foods on the main outcome, weight gain $(\mathrm{g} / \mathrm{kg}$ per $\mathrm{d})$. To detect a $10 \%$ difference with a standard deviation of $0.7 \mathrm{~g} / \mathrm{kg}$ per $\mathrm{d}$, a sample size of forty-nine in each group was required to achieve $80 \%$ power $(\alpha=0.05$, two-sided). To allow for dropouts, a total of 120 children were enrolled ${ }^{(29)}$. Initial recruitment was of 0.5-18-year-old children. After recruiting fiftyfour children, the recruitment criterion for age was adjusted to enrol only 6-59-month-old children due to limited funding and on request of the funding agency (UNICEF). The final sample was comprised of the fiftyfour children aged up to 18 years and 120 children aged 6-59 months.

\section{Eligibility and nutritional markers}

The eligibility criteria for enrolment in the present study were children aged 0.5-18 years with non-complicated acute malnutrition, defined as weight-for-height $Z$-score (WHZ) or BMI-for-age $Z$-score (BAZ) of $\leq-2 \cdot 8$. Children younger than 60 months were also re-enrolled based on mid-upper-arm circumference (MUAC) of $\leq 115 \mathrm{~mm}$ and/or presence of nutritional oedema ${ }^{(29)}$. Children with complications affecting food intake or participating in other clinical trials were not eligible. Personal and sociodemographic information was obtained from caregivers using questionnaires. Age was obtained from birth growth monitoring cards, or by asking the caregiver if the card was not available. Current breast-feeding status was reported by interviewing caregivers using a structured questionnaire.

Nutritional markers were assessed by anthropometric measures (weight, length/height, MUAC, skinfolds) using standard equipment as described in Sigh et al. ${ }^{(29)}$. Anthropometric $Z$-scores, namely WHZ or BAZ (wasting), weight-for-age $Z$-score (WAZ; underweight) and heightfor-age $Z$-score (HAZ; stunting), were calculated from the WHO Growth Standards 2006 for children aged 6-59 months $^{(31)}$ and the WHO Growth References 2007 for children aged 5-19 years ${ }^{(32)}$. The mid-upper-arm fat percentage and muscle area were calculated according to Rolland-Cachera et al. ${ }^{(33)}$ using MUAC and triceps skinfold measures, which were measured to nearest $0.1 \mathrm{~mm}$ using a Holtain skinfold calliper.

\section{Blood sampling}

Four millilitres of venous whole blood from the cubital vein were drawn using a sterile $23 \mathrm{G} / 25 \mathrm{G} \times 3 / 4$ " scalp vein set (Vinahankook Medical Supplies Co., Hanoi, Vietnam) or a sterile $24 \mathrm{G}$ IV cannula needle with catheter and injection valve (Harsoria ${ }^{\mathrm{TM}}$, Gurgaon, India) by trained health staff. Two millilitres of whole blood were collected in $2 \times 2 \mathrm{ml}$ EDTA-coated vacutainers and $2 \mathrm{ml}$ of whole blood were collected in a $6 \mathrm{ml}$ trace-element Sodium $\mathrm{NH}$ Heparin vacutainer (VACUETTE ${ }^{\circledR}$; Greiner Bio-One International GmbH, Frickenhausen, Germany). One EDTA vacutainer was used to perform dried blood spots to measure PUFA and blood cell count analysis. Haemoglobinopathies were analysed using the second EDTA vacutainer and inflammation status was analysed using the sodium heparin vacutainer.

\section{Dried blood spots and fatty acid analysis}

Dried blood spots were performed using the laboratory facilities at the National Pediatric Hospital, following validated procedures ${ }^{(34)}$. Dried blood spots were made 
by applying $40 \mu \mathrm{l}$ of EDTA whole blood on pre-treated chromatography paper (Grade 3MM, Whatman Ltd; Danish supplier: Frederiksen Scientific A/S, Ølgod, Denmark). The samples were dried at room temperature and stored in a polypropylene 'Ziplock' bag at $4{ }^{\circ} \mathrm{C}$ for a maximum of 2 months prior to shipment to the University of Waterloo in Canada for fatty acid analysis $^{(34,35)}$. The samples were analysed on a Varian 3900 gas chromatograph equipped with a DB-FFA $15 \mathrm{~m} \times$ $0.10 \mathrm{~mm}$ capillary column coated with a $0 \cdot 10 \mu \mathrm{m}$ film of nitroterephthalic-acid-modified polyethylene glycol (J\&W Scientific; Agilent Technologies, Mississauga, ON, Canada) using hydrogen as the carrier gas ${ }^{(35-38)}$. The eluted fatty acid methyl ester peaks were identified by comparing retention times with an external standard (GLC-246; Nu Chek Prep Inc., Elysian, MN, USA) ${ }^{(39)}$. Fatty acid data are expressed as relative percentage of all fatty acids ( $\%$ FA) and as fatty acid concentration ( $\mu$ g per $100 \mu \mathrm{g}$ whole blood).

The inter- and intra-assay CV for the dried blood spot fatty acid analyses were similar to values previously reported for wet whole-blood analyses ${ }^{(40)}$. For the fatty acids reported, all the inter-assay CV were below $7 \%$ and all the intra-assay CV were below $3 \%$, with higher $\mathrm{CV}$ being associated with the fatty acids with relatively low abundance (i.e. docosapentaenoic acid (22:5n-6; DPA) and mead acid (20:3n-9; MA)).

\section{Definition of essential fatty acid deficiency}

LOw LA and ALA are reflected in a ratio between their longchain metabolites. The ratios MA:AA and $n-6$ DPA:DHA are commonly used biomarkers of essential fatty acid deficiencies $^{(41,42)}$. For whole blood, deficiency levels for these markers have not been defined. However, it has been proposed that in whole blood, MA:AA $>0.02$ indicates very low $n-6$ PUFA status and $n-6$ DPA:DHA $>0.2$ and total $n-6$ : $n-3$ PUFA $>10 \cdot 5$ indicate very low $n-3$ PUFA status ${ }^{(16)}$. We applied these cut-off values to define low PUFA.

\section{Assessment of health markers}

The markers assessed for health status were Hb concentration (for anaemia), blood cell counts (overall health status), $\mathrm{Hb}$ variations (for haemoglobinopathies), the presence of HIV (for HIV status), C-reactive protein (CRP; acute inflammation marker) and $\alpha_{1}$-acid glycoprotein (AGP; progressed inflammation marker). Approximately $300 \mu$ EDTA whole blood was used to assess blood cell counts, including $\mathrm{Hb}$ concentration, using a haematology analyser (Sysmex Kx-21, ID no. NP021 and NP023; Sysmex Corporation, Kobe, Japan) at the National Pediatric Hospital. Anaemia status was diagnosed based on $\mathrm{Hb}$ concentration using cut-off values recommended by $\mathrm{WHO}^{(43)}$. The presence of haemoglobinopathies (only in the 6-59-month-old children) was analysed using electrophoreses (Minicap; SEBIA, Lisses, France). Samples were analysed at the National Pediatric Hospital. Following a disruption of the capacity for analysing at the hospital, five samples were analysed at the Pasture Institute, Phnom Penh, using the same method and analysis protocol. Normal Hb was classified for 0-23-month-old children as $\mathrm{HbA}>70 \%$, $\mathrm{HbF} \leq 30 \%, \mathrm{HbA} 2<2 \%$; and for 24-59-month-old children as $\mathrm{HbA}>95.5 \%, \mathrm{HbA} 2=2 \cdot 0-3.5 \%, \mathrm{HbF}<5 \%$. All other $\mathrm{Hb}$ variations were classified as haemoglobinopathies, except HbE/heterozygote, which was classified as $\mathrm{HbE} \sim 20-30 \%{ }^{(44)}$. Complete blood counts and haemoglobinopathies were analysed one sample at a time and no certified standard was available. Therefore, no interand intra-assay CV were available.

HIV status was self-reported via a questionnaire asking if the child had been tested and, if so, the result of the test. All HIV-positive children in the study received treatment at the National Pediatric Hospital. CRP and AGP were measured in a combined sandwich ELISA method in duplicate ${ }^{(45)}$. The control samples' ( $n$ 40) inter-assay CV were $5.8 \%$ (CRP) and $8.1 \%$ (AGP). Inflammation was indicated by CRP $>5 \mathrm{mg} / \mathrm{l}$ and/or $\mathrm{AGP}>1 \mathrm{~g} / \mathrm{l}^{(46)}$.

\section{Statistical analysis}

All data, except blood analyses, were entered directly into tablets and uploaded to an online software system (Kobotoolbox) or double entered into EpiData version 3.1 (The EpiData Association, Odense, Denmark). Statistical analyses were conducted using the software R studio (version 3.4.0 for Windows). Models were assessed by visual residuals and normal probability plots inspection. Non-normal distributed models were $\log _{10}$ transformed. Descriptive statistics are presented as percentages and frequencies, means and standard deviations, or medians and interquartile ranges. All analyses, not pre-including age, are ageadjusted owing to large age variations in the study. To analyse correlations between PUFA and nutritional and health status markers, a simple or multiple linear regression was applied adjusted for age, sex and breast-feeding due to the well-known effects of breast-feeding on PUFA $^{(16,47-50)}$. The analyses are reported as regression coefficients $(\beta)$ with $95 \%$ confidence intervals and $P$ values. Comparisons in cell counts between health markers were assessed using ANCOVA adjusted for age and sex with the Emmeans R package, which takes multiple comparisons into consideration. Statistical significance was defined as $P<0.05$. $P$ values were not adjusted for false discovery rates, as the correlation between PUFA and markers of nutrition status was consistent across variables, meaning the $P$ values would be overcorrected if adjusted using the traditional Bonferroni method, resulting in a lower correlation factor and hence increased risk of type 2 errors. 
Table 1 Characteristics of the Cambodian children with acute malnutrition from the SAM trial conducted from September 2015 until January 2017 (mean and range; means and standard deviations; percentages and numbers; medians and interquartile ranges)

\begin{tabular}{|c|c|c|}
\hline \multirow[b]{2}{*}{ Characteristic } & \multicolumn{2}{|c|}{ Children with acute malnutrition $(n 174)$} \\
\hline & Mean, $\%$ or median & Min-max, SD, $n$ or IQR \\
\hline Age (years) $\dagger$, mean and min-max & $4 \cdot 1$ & $0 \cdot 4-16 \cdot 6$ \\
\hline \multicolumn{3}{|l|}{ Sex, $\%$ and $n$} \\
\hline Female & 35 & 60 \\
\hline Currently breast-feeding, $\%$ and $n$ & 35 & 61 \\
\hline \multicolumn{3}{|l|}{ Nutritional status } \\
\hline Wasting Z-scoreł, mean and SD & -3.0 & 0.8 \\
\hline WAZ§, mean and SD & -3.3 & 0.9 \\
\hline Body weight $(\mathrm{kg})$, mean and SD & $11 \cdot 2$ & $7 \cdot 8$ \\
\hline $\mathrm{HAZ}$, mean and SD & $-2 \cdot 4$ & 1.3 \\
\hline Height $(\mathrm{cm})$, mean and SD & 88 & 26 \\
\hline MUAC $(\mathrm{cm})$, mean and SD & $12 \cdot 8$ & 24 \\
\hline $\mathrm{SAM} \|, \%$ and $n$ & 58 & 101 \\
\hline MAM $\|, \%$ and $n$ & 36 & 63 \\
\hline Mid-upper-arm fat (\%), mean and SD & 31 & 9 \\
\hline Mid-upper-arm muscle area $\left(\mathrm{cm}^{2}\right)$, mean and SD & $9 \cdot 6$ & 4.5 \\
\hline \multicolumn{3}{|l|}{ Health status } \\
\hline Oedema, $\%$ and $n$ & 0.0 & 0 \\
\hline $\mathrm{Hb}(\mathrm{g} / \mathrm{dl})$, mean and SD & $11 \cdot 1$ & 1.5 \\
\hline Anaemiaq, $\%$ and $n$ & $52 \cdot 3$ & 91 \\
\hline \multicolumn{3}{|l|}{ Haemoglobinopathy††, \% and $n$} \\
\hline $\mathrm{Hb}$ normal & $65 \cdot 1$ & 69 \\
\hline $\mathrm{Hb}$ disorders & 34.9 & 37 \\
\hline $\mathrm{HbE} /$ heterozygote & 20.8 & 22 \\
\hline \multicolumn{3}{|l|}{ Infection, $\%$ and $n$} \\
\hline HIV positive & 20 & 35 \\
\hline \multicolumn{3}{|l|}{ Inflammation markers } \\
\hline Plasma CRP (mg/l), median and IQR & 0.6 & $0 \cdot 2-2 \cdot 3$ \\
\hline Plasma CRP (>5 mg/l), \% and $n$ & 16 & 27 \\
\hline Plasma AGP ( $\mathrm{g} / \mathrm{l})$, median and IQR & 0.7 & $0.5-1.3$ \\
\hline Plasma AGP $(>1 \mathrm{~g} / \mathrm{l}), \%$ and $n$ & 35 & 61 \\
\hline 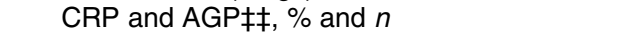 & 12 & 20 \\
\hline \multicolumn{3}{|l|}{ Blood counts $\S$, mean and SD } \\
\hline Erythrocytes $\left(\times 10^{12} / \mathrm{l}\right)$ & 4.6 & 0.7 \\
\hline Platelets $\left(\times 10^{9} / \mathrm{I}\right)$ & 406 & 161 \\
\hline Leucocytes $\left(\times 10^{9} / l\right)$ & $11 \cdot 7$ & 4.5 \\
\hline Neutrophils $\left(\times 10^{9} / I\right)$ & 4.4 & $2 \cdot 2$ \\
\hline Lymphocytes $\left(\times 10^{9} / \mathrm{I}\right)$ & $6 \cdot 1$ & 3.1 \\
\hline Monocytes $\left(\times 10^{9} / \mathrm{I}\right)$ & 0.9 & 0.5 \\
\hline Eosinophils $\left(\times 10^{9} / \mathrm{l}\right)$ & 0.3 & 0.4 \\
\hline Total cells \|\|$\left(\times 10^{12} / l\right)$ & 4.8 & 0.7 \\
\hline
\end{tabular}

IQR, interquartile range; WAZ, weight-for-age Z-score; HAZ, height-for-age Z-score; MUAC, mid-upper-arm circumference; SAM, severe acute malnutrition; MAM, moderate acute malnutrition; CRP, C-reactive protein; AGP, $\alpha_{1}$-acid glycoprotein. $\dagger n 173$, missing exact age and sex from one child.

¥Measured using weight-for-height Z-score (WHZ) or BMl-for-age Z-score (BAZ) as an indication of wasting.

$\S n 146$, missing specific birthdate for twenty-eight children.

$\|$ Among children aged $\leq 59$ months: SAM defined as either $\mathrm{WHZ}<-3$ or MUAC $<115 \mathrm{~mm}$; MAM defined as either WHZ between -2 and -3 or MUAC $=115-125 \mathrm{~mm}$. Among children aged $>60$ months: SAM defined as either BAZ $<-3$ or MUAC $<129 \mathrm{~mm}$ (children aged 5-9 years), MUAC $<160 \mathrm{~mm}$ (children aged 10-14 years), MUAC $<190 \mathrm{~mm}$ (children aged 15-18 years); MAM defined as either BAZ between -2 and -3 or $M U A C=129-154 \mathrm{~mm}$ (children aged 5-9 years), MUAC $=160-185 \mathrm{~mm}$ (children aged 10-14 years), MUAC $=190-215 \mathrm{~mm}$ (children aged $15-18$ years).

TAnaemia cut-off is age- and sex-dependent.

††Haemoglobinopathies were only assessed in the 6-59-month-old children, $n 106$ out of the total 174; thus the percentage is calculated based on the $n$ of the actual sampling.

犃efined as CRP $>5 \mathrm{mg} / \mathrm{l}$ and $\mathrm{AGP}>1 \mathrm{~g} / \mathrm{l}$

$\S \S n 163$, data on blood cell counts were missing for ten children.

\|IIErythrocytes, leucocytes and platelets combined.

\section{Results}

\section{Participants}

Out of 609 children screened, 174 (28.6\%) were eligible for enrolment (Table 1). Their mean age was 4.1 years and their mean wasting $Z$-score was $-3 \cdot 0$. The anaemia prevalence was $52 \%, \sim 35 \%$ of the children had haemoglobinopathies and $20 \%(n 35)$ were HIV positive. Median levels of inflammation markers were $0.6 \mathrm{mg} / 1$ (CRP) and $0.7 \mathrm{~g} / \mathrm{l}$ (AGP), reflecting an inflammation prevalence of 16 and $35 \%$ according to CRP and AGP, respectively. 
Table 2 Whole-blood fatty acid composition of the malnourished Cambodian children from the SAM trial conducted from September 2015 until January 2017 (means and standard deviations)

\begin{tabular}{|c|c|c|c|}
\hline \multirow[b]{2}{*}{ Whole-blood fatty acid (\% FA) } & & \multicolumn{2}{|c|}{$\begin{array}{l}\text { Children with } \\
\text { acute } \\
\text { malnutrition } \\
(n 174)\end{array}$} \\
\hline & & Mean & SD \\
\hline \multicolumn{4}{|l|}{ SFA } \\
\hline Lauric acid & $12: 0$ & 0.5 & 0.5 \\
\hline Myristic acid & $14: 0$ & $1 \cdot 7$ & 0.9 \\
\hline Palmitic acid & $16: 0$ & $27 \cdot 4$ & $2 \cdot 1$ \\
\hline Margaric acid & $17: 0$ & 0.4 & 0.1 \\
\hline Steric acid & $18: 0$ & 11.9 & $2 \cdot 2$ \\
\hline Arachidic acid & $20: 0$ & 0.3 & 0.1 \\
\hline Behenic acid & $22: 0$ & 0.8 & 0.2 \\
\hline Tricosylic acid & $23: 0$ & 0.1 & 0.1 \\
\hline Lignoceric acid & $24: 0$ & 1.4 & 0.4 \\
\hline Total SFA & & 45.5 & $3 \cdot 4$ \\
\hline \multicolumn{4}{|l|}{ MUFA } \\
\hline Palmitoleic acid & $16: 1 n-7$ & $1 \cdot 7$ & 0.7 \\
\hline Vaccenic acid & $18: 1 n-7$ & $1 \cdot 7$ & 0.4 \\
\hline Oleic acid & $18: 1 n-9$ & 23.4 & 3.6 \\
\hline Gondoic acid & $20: 1 n-9$ & 0.3 & 0.1 \\
\hline Erucic acid & $22: 1 n-9$ & 2.4 & 1.0 \\
\hline Nervonic acid & $24: 1 n-9$ & 1.5 & 0.4 \\
\hline Total MUFA & & $31 \cdot 1$ & 3.7 \\
\hline \multirow{2}{*}{\multicolumn{4}{|c|}{$n-6$ PUFA }} \\
\hline & & & \\
\hline Linoleic acid (LA) & $18: 2 n-6$ & $13 \cdot 2$ & $2 \cdot 7$ \\
\hline$\gamma$-Linoleic acid & $18: 3 n-6$ & 0.1 & 0.1 \\
\hline Eicosadienoic acid & $20: 2 n-6$ & 0.2 & 0.0 \\
\hline Dihomo- $\gamma$-linolenic acid & $20: 3 n-6$ & 0.7 & $0 \cdot 2$ \\
\hline Arachidonic acid (AA) & $20: 4 n-6$ & 3.6 & 1.3 \\
\hline Docosadienoic acid & $22: 2 n-6$ & 0.1 & 0.0 \\
\hline Adrenic acid & $22: 4 n-6$ & 0.4 & 0.1 \\
\hline Docosapentaenoic acid ( $n-6$ DPA) & $22: 5 n-6$ & 0.2 & 0.1 \\
\hline Total $n-6$ PUFA & & $18 \cdot \overline{6}$ & 3.5 \\
\hline \multicolumn{4}{|l|}{$n-3$ PUFA } \\
\hline$\alpha$-Linolenic acid (ALA) & $18: 3 n-3$ & 0.2 & 0.1 \\
\hline EPA & $20: 5 n-3$ & 0.1 & 0.1 \\
\hline Docosapentaenoic acid ( $n$-3 DPA) & $22: 5 n-3$ & 0.2 & 0.1 \\
\hline DHA & $22: 6 n-3$ & 0.8 & 0.3 \\
\hline Total $n$-3 PUFA & & 1.5 & 0.5 \\
\hline Total PUFA & & $20 \cdot 1$ & 3.7 \\
\hline Total fatty acid concentration $\dagger$ & & 349 & 131 \\
\hline \multicolumn{4}{|l|}{ Deficiency biomarkers } \\
\hline MA:AA & & 0.02 & 0.02 \\
\hline$n-6$ DPA:DHA & & 0.4 & 0.2 \\
\hline$n-6: n-3$ PUFA & & $13 \cdot 5$ & 3.9 \\
\hline
\end{tabular}

$\%$ FA, percentage of total fatty acids.

†Total fatty acid concentration is expressed as $\mu \mathrm{g} / 100 \mu \mathrm{l}$ whole blood.

\section{Whole-blood PUFA and very low PUFA status}

The relative contribution in whole blood was $13.2 \% \mathrm{FA}$ for LA and was $0.2 \%$ FA for ALA (Table 2 ). The mean relative contribution for MA was $0 \cdot 1 \% \mathrm{FA}$ and this was reflected in a high MA:AA ratio, indicating very low $n-6$ PUFA status in the children according to the predefined cut-off (MA:AA > 0.02). Based on the predefined cut-offs ( $n-6$ DPA:DHA $>0 \cdot 2$ and total $n-6: n-3$ PUFA $>10 \cdot 5), 68 \%$ of the children appeared to have very low $n-3$ PUFA status. We estimated that $14 \%$ of the children may actually be both $n-6$ and $n-3$ deficient (MA:AA $>0 \cdot 02, n-6$ DPA:DHA $>0 \cdot 2$ and total $n-6: n-3$ PUFA $>10 \cdot 5)$ based on these cut-offs.

\section{Associations between whole-blood PUFA and markers of nutritional status}

HAZ was significantly and positively correlated with total PUFA $(\beta=0.57 \%$ FA; $95 \%$ CI $0.14,1.00 \%$ FA), LA $(\beta=0.33 \%$ FA; $95 \%$ CI $0.02,0.64 \%$ FA), AA $(\beta=0.16 \%$ FA; $95 \%$ CI $0.01,0.31 \%$ FA) and total $n-6$ LCPUFA $(\beta=0.20 \%$ FA; $95 \%$ CI $0.02,0.38 \%$ FA; Table 3$)$. The estimates indicated that an increase in HAZ of 1 was correlated with a $\sim 0.6 \%$ FA-point increase in total PUFA. A similar association was found for absolute age-adjusted height. Height was also significantly positively associated with whole-blood DHA and n-3 LCPUFA (Table 4). A $6 \mathrm{~cm}$ increase in height was correlated with a $\sim 0.7 \%$ FA-point increase in DHA (Table 4). A $50 \mathrm{~cm}$ increase in height was associated with a $-0.01 \%$ FApoint decrease in MA:AA and hence with a decreased risk of $n-6$ essential fatty acid deficiencies.

MUAC was not significantly correlated with any of the PUFA measures (see online supplementary material, Supplemental Tables S1 and S2). However, separating MUAC into fat percentage and muscle area showed that a low mid-upper-arm fat percentage was associated with high AA, n-6 LCPUFA and n-3 LCPUFA (Tables 3 and 4), and a high mid-upper-arm muscle area was positively associated with AA and $n$-6 LCPUFA (Table 3 ).

Wasting Z-score and WAZ were not associated with any PUFA, but high body weight was associated with high EPA (20:5n-3; see online supplementary material, Supplemental Tables S1 and S2).

\section{Associations between markers of bealth challenges and whole-blood PUFA}

$\mathrm{Hb}$ concentration was not correlated with overall PUFA, $n-6$ PUFA, $n-3$ PUFA or either of the markers of low PUFA, MA:AA or $n-6$ DPA:DHA (Tables 3 and 4). Also, no associations were observed for anaemia (see online supplementary material, Supplemental Tables S1 and S2). However, the presence of haemoglobinopathies was associated with high MA:AA and a non-statistically significant tendency for low total PUFA ( $\beta=-1.34 \%$ FA-points; $95 \%$ CI $-2.72,0.04 \%$ FA-points; Table 3). An age-stratified analysis showed a $1.39 \%$ FA-point (95\% CI $-2.78,-0.00 \%$ FA-point, $n$ 103) lower total PUFA in 6-59-month-old children with haemoglobinopathies than in the children without haemoglobinopathies.

HIV infection and high AGP concentration were associated with high MA:AA (Table 3). No associations were observed between CRP and any of the PUFA (Tables 3 and 4).

Unexpectedly, total whole-blood cell count was not associated with any of the PUFA measures. Furthermore, no correlations were observed for total count of leucocytes or erythrocytes. However, high platelet count was associated with high total PUFA and LA 


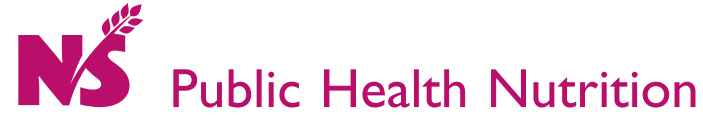

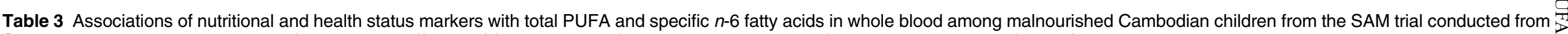
September 2015 until January 2017 (regression coefficients $(\beta)$ and $95 \%$ confidence intervals adjusted for age, sex and breast-feeding)

\begin{tabular}{|c|c|c|c|c|c|c|c|c|c|c|}
\hline & \multirow{2}{*}{\multicolumn{2}{|c|}{ Total PUFA† }} & \multicolumn{8}{|c|}{$n-6$ PUFA } \\
\hline & & & \multicolumn{2}{|r|}{ LA† } & \multicolumn{2}{|r|}{$\mathrm{AA} \dagger$} & \multicolumn{2}{|c|}{$n-6$ LCPUFA† } & \multicolumn{2}{|c|}{ MA:AA†,‡ } \\
\hline & $\beta$ & $95 \% \mathrm{Cl}$ & $\beta$ & $95 \% \mathrm{Cl}$ & $\beta$ & $95 \% \mathrm{Cl}$ & $\beta$ & $95 \% \mathrm{Cl}$ & $\beta$ & $95 \% \mathrm{Cl}$ \\
\hline \multicolumn{11}{|l|}{ Nutritional status } \\
\hline Wasting Z-score§, || (n 168) & -0.06 & $-0.70,0.83$ & $-0 \cdot 13$ & $-0.43,0.68$ & 0.02 & $-0.29,0.24$ & -0.03 & $-0.36,0.29$ & -0.24 & $-4 \cdot 82,4 \cdot 34$ \\
\hline HAZ $\|(n 168)$ & 0.57 & $0.14,1.00^{\star}$ & 0.33 & $0.02,0.64^{*}$ & 0.16 & $0.01,0.31^{\star}$ & 0.20 & $0.02,0.38^{*}$ & -1.85 & $-4.37,0.66$ \\
\hline Height $(m)(n 168)$ & $15 \cdot 21$ & $6 \cdot 23,4 \cdot 19^{*}$ & $7 \cdot 86$ & $1 \cdot 26,14 \cdot 46^{*}$ & 4.92 & $1 \cdot 78,8 \cdot 05^{\star}$ & $6 \cdot 10$ & $2 \cdot 23,9 \cdot 97^{\star}$ & -16 & $-109,-4^{*}$ \\
\hline Mid-upper-arm fat $(\%)(n 168)$ & -0.05 & $-0.11,0.03$ & -0.00 & $-0.28,0.00$ & -0.03 & $-0.05,-0.00^{*}$ & -0.03 & $-0.06,-0.00^{\star}$ & -0.05 & $-0.44,0.35$ \\
\hline Mid-upper-arm muscle area $\left(\mathrm{cm}^{2}, \times 10\right)(n 168)$ & 1.83 & $-0.83,4.49$ & 0.21 & $-1 \cdot 73,2 \cdot 14$ & $1 \cdot 22$ & $0.31,2 \cdot 13^{*}$ & 1.49 & $0.36,2 \cdot 61^{*}$ & $-7 \cdot 43$ & $-22 \cdot 75,7 \cdot 89$ \\
\hline \multicolumn{11}{|l|}{ Health status } \\
\hline $\mathrm{Hb}(\mathrm{g} / \mathrm{dl})(n 165)$ & 0.24 & $-0.17,0.64$ & 0.14 & $-0.17,0.43$ & 0.07 & $-0.07,0.21$ & 0.09 & $-0.09,0.26$ & -2.04 & $-4 \cdot 36,0.27$ \\
\hline Haemoglobinopathy (yes v. no) (n 105) & -1.34 & $-2 \cdot 72,0.04$ & $-1 \cdot 01$ & $-2 \cdot 05,0.03$ & $-0 \cdot 36$ & $-0.90,0.17$ & -0.33 & $-0.97,0.31$ & 8.53 & $0.12,16.94^{*}$ \\
\hline \multicolumn{11}{|l|}{ Infection } \\
\hline HIV infection, (yes v. no) (n66) & -0.27 & $-3.52,2.98$ & -1.07 & $-3.37,1.23$ & 0.35 & $-0.66,1.35$ & 0.75 & $-0.56,2.06$ & 24.82 & $8 \cdot 11,41 \cdot 54^{*}$ \\
\hline \multicolumn{11}{|l|}{ Inflammation markers } \\
\hline CRP $\left(\mathrm{mg} / \mathrm{l}, \times 10^{-4}\right)(n 159)$ & -19 & $-88,49$ & -17 & $-66,32$ & 1.12 & $-23 \cdot 33,25 \cdot 58$ & 0.13 & $-0 \cdot 23,1$ & 133 & $-232,499$ \\
\hline AGP $\left(\mathrm{g} / \mathrm{l}, \times 10^{2}\right)(n 159)$ & -598 & $-1299,90$ & -432 & $-920,57$ & -105 & $-351,141$ & -99 & $-403,206$ & 6640 & $3102,10177^{*}$ \\
\hline \multicolumn{11}{|l|}{ Blood counts } \\
\hline Erythrocytes $\left(\times 10^{15} / I\right)(n 165)$ & 0.56 & $-2 \cdot 30,3 \cdot 43$ & $13 \cdot 16$ & $-48.37,74.69$ & 0.55 & $-29 \cdot 04,30 \cdot 14$ & $0 \cdot 30$ & $-36 \cdot 23,36 \cdot 83$ & -4.49 & $-9.36,3.77$ \\
\hline Platelets $\left(\times 10^{12} / l\right)(n 165)$ & 945 & $299,1590^{*}$ & 0.38 & $0.12,0.64^{*}$ & 0.10 & $-0.03,0.22$ & 0.12 & $-0.03,0.28$ & -924 & $-2050,202$ \\
\hline 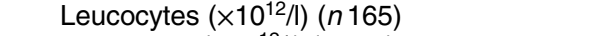 & $5 \cdot 29$ & $-1 \cdot 23,21 \cdot 83$ & $-3 \cdot 20$ & $-7 \cdot 44,13 \cdot 84$ & 0.89 & $-4 \cdot 23,6 \cdot 00$ & 1.09 & $-5 \cdot 23,7 \cdot 41$ & $-23 \cdot 20$ & $-51.99,4.35$ \\
\hline Monocytes $\left(\times 10^{12} / /\right)(n 165)$ & 165 & $46,284^{*}$ & 84 & $-3,171$ & 51 & $10,93^{*}$ & 63 & $12,114^{\star}$ & -179 & $-522,164$ \\
\hline Neutrophils $\left(\times 10^{12} / l\right)(n 165)$ & -64 & $-975,847$ & -1.04 & $-20 \cdot 40,18.33$ & -1.06 & $-10 \cdot 37,8.25$ & $-1 \cdot 23$ & $-12 \cdot 72,10 \cdot 26$ & -1238 & $-2789,313$ \\
\hline Total cellsף $\left(\times 10^{12} / l\right)(n 165)$ & 68 & $-23,359$ & 0.15 & $-0.46,0.75$ & 0.01 & $-0.28,0.30$ & 0.01 & $-0.35,0.37$ & -484 & $-978,10$ \\
\hline
\end{tabular}

LA, linoleic acid; AA, arachidonic acid; LCPUFA, long-chain PUFA; MA, mead acid; HAZ, height-for-age Z-score; CRP, C-reactive protein; AGP, $\alpha_{1}$-acid glycoprotein.

${ }^{*} P<0.05$.

†For blood counts only, results are given as $\times 10^{-4}$ for total PUFA, $\times 10^{3}$ for LA and AA, $\times 10^{2}$ for $n-6$ LCPUFA and $\times 10^{-2}$ for MA:AA to present meaningful results.

FFor nutritional status and health status markers, results (except blood counts) are given as $\times 10^{3}$ to present meaningful results.

$\S$ Wasting $Z$-score is weight-for-height $Z$-score for children aged $<5$ years and BMI-for-age $Z$-score $B A Z$ for children aged $\geq 5$ years.

Adjusted only for breast-feeding.

Frythrocytes, leucocytes and platelets combined. 
Table 4 Associations of nutritional and health status markers with specific $n$ - 3 fatty acids in whole blood among malnourished Cambodian children from the SAM trial conducted from September 2015 until January 2017 (regression coefficients $(\beta)$ and $95 \%$ confidence intervals adjusted for age, sex and breast-feeding)

\begin{tabular}{|c|c|c|c|c|c|c|c|c|}
\hline & \multicolumn{8}{|c|}{$n$-3 PUFA } \\
\hline & \multicolumn{2}{|c|}{ EPA†,‡ } & \multicolumn{2}{|c|}{ DHA } & \multicolumn{2}{|c|}{$n-3$ LCPUFA†, $\ddagger$} & \multicolumn{2}{|c|}{ n-6 DPA:DHA†,‡ } \\
\hline & $\beta$ & $95 \% \mathrm{Cl}$ & $\beta$ & $95 \% \mathrm{Cl}$ & $\beta$ & $95 \% \mathrm{Cl}$ & $\beta$ & $95 \% \mathrm{Cl}$ \\
\hline \multicolumn{9}{|l|}{ Nutritional status } \\
\hline Wasting Z-score§, $\|(n$ 168) & $5 \cdot 7$ & $-5 \cdot 8,17 \cdot 2$ & 7.4 & $-66 \cdot 5,1 \cdot 3$ & $-48 \cdot 3$ & $-152,430$ & -8.4 & $-47 \cdot 3,30 \cdot 6$ \\
\hline $\mathrm{HAZ} \|(n 168)$ & $5 \cdot 2$ & $-1 \cdot 2,11 \cdot 7$ & $25 \cdot 3$ & $-16 \cdot 5,7 \cdot 1$ & 30.5 & $-29 \cdot 0,89 \cdot 1$ & $-7 \cdot 7$ & $-29 \cdot 8,14 \cdot 4$ \\
\hline Height $(m)(n 168)$ & 175 & $-378,313$ & 1111 & $226,1995^{\star}$ & 1283 & $24,2541^{*}$ & -494 & $-962,-27^{\star}$ \\
\hline Mid-upper-arm fat (\%) (n 168) & -0.5 & $-1.5,0.6$ & $-4 \cdot 8$ & $-11.5,1.9$ & -9.54 & $-18 \cdot 9,-0 \cdot 17^{*}$ & $3 \cdot 2$ & $-0.3,6 \cdot 7$ \\
\hline Mid-upper-arm muscle area $\left(\mathrm{cm}^{2}, \times 10\right)(n 168)$ & 29 & $-11,69$ & 186 & $-72,444$ & 164 & $-202,530$ & -95 & $-231,41$ \\
\hline \multicolumn{9}{|l|}{ Health status } \\
\hline $\mathrm{Hb}(\mathrm{g} / \mathrm{dl})(n 165)$ & $-4 \cdot 6$ & $-10 \cdot 7,1 \cdot 4$ & 4.7 & $-34 \cdot 7,44 \cdot 1$ & $2 \cdot 1$ & $-3.5,7.67$ & $7 \cdot 0$ & $-13 \cdot 8,27 \cdot 8$ \\
\hline Haemoglobinopathy (yes v. no) $(n 105)$ & 1.4 & $-21 \cdot 1,23 \cdot 8$ & -48 & $-201,105$ & -0.7 & $-18 \cdot 1,16 \cdot 7$ & $6 \cdot 4$ & $-55 \cdot 4,68 \cdot 2$ \\
\hline \multicolumn{9}{|l|}{ Infection } \\
\hline $\begin{array}{l}\text { HIV infection (yes } v . \text { no) (n66) } \\
\text { Inflammation markers }\end{array}$ & 4.9 & $-37 \cdot 6,47 \cdot 5$ & -5 & $-256,245$ & $-29 \cdot 1$ & $-496,440$ & $18 \cdot 5$ & $-158,195$ \\
\hline CRP $\left(\mathrm{mg} / \mathrm{l}, \times 10^{-4}\right)(n 159)$ & -157 & $-1,897$ & -1367 & $-8136,5401$ & -4676 & $-14234,4881$ & -39 & $-3653,3574$ \\
\hline AGP $\left(\mathrm{g} / \mathrm{l}, \times 10^{2}\right)(n 159)$ & 914 & $-11556,9727$ & -34780 & $-102858,33299$ & -78210 & $-174094,17682$ & 8272 & $-28150,44694$ \\
\hline \multicolumn{9}{|l|}{ Blood counts } \\
\hline Erythrocytes $\left(\times 10^{15} / l\right)(n 165)$ & $-7 \cdot 2$ & $-26 \cdot 2,11 \cdot 8$ & $2 \cdot 6$ & $-5 \cdot 7,10 \cdot 8$ & 4.3 & $-7 \cdot 4,16 \cdot 0$ & -1.6 & $-7 \cdot 2,4 \cdot 0$ \\
\hline Platelets $\left(\times 10^{12} / I\right)(n 165)$ & 3645 & $-712,8003$ & 0.02 & $-0.02,0.05$ & 0.02 & $-0.03,0.07$ & -625 & $-1914,664$ \\
\hline Leucocytes $\left(\times 10^{12} / l\right)(n 165)$ & $56 \cdot 7$ & $-53 \cdot 1,16 \cdot 6$ & 0.6 & $-0 \cdot 9,2 \cdot 0$ & 0.4 & $-1 \cdot 7,2 \cdot 4$ & -12.5 & $-44 \cdot 8,19 \cdot 8$ \\
\hline Monocytes $\left(\times 10^{12} / /\right)(n 165)$ & 818 & $-509,2144$ & 19 & $8,31^{*}$ & 18 & $1,34^{*}$ & -273 & $-663,116$ \\
\hline Neutrophils $\left(\times 10^{12} / l\right)(n 165)$ & 863 & 5183,6910 & 0.5 & $-2 \cdot 1,3 \cdot 1$ & $1 \cdot 2$ & $-2 \cdot 5,4 \cdot 8$ & -984 & $-2757,789$ \\
\hline Total cellsף $\left(\times 10^{12} / l\right)(n 165)$ & -629 & $-2561,1304$ & 0.03 & $-0.05,0.11$ & 0.04 & $-0.07,0.16$ & -183 & $-752,385$ \\
\hline
\end{tabular}

LCPUFA, Iong-chain PUFA; DPA, docosapentanoic acid; CRP, C-reactive protein; AGP, $\alpha_{1}$-acid glycoprotein.

${ }^{*} P<0.05$.

†For blood counts only, results are total given as $\times 10^{-2}$ for EPA, $\times 10^{2}$ for $n-3$ LCPUFA and $\times 10^{-3}$ for $n-6$ DPA:DHA to present meaningful results.

fFor nutritional status and health status markers, results (except blood counts) are given as $\times 10^{3}$ to present meaningful results.

§Wasting $Z$-score is weight-for-height $Z$-score for children aged $<5$ years and BMl-for-age $Z$-score BAZ for children aged $\geq 5$ years.

$\|$ Adjusted only for breast-feeding.

ףErythrocytes, leucocytes and platelets combined. 
Table 5 Comparison of blood counts in the presence or anaemia, HIV, inflammation or haemoglobinopathies among malnourished Cambodian children from the SAM trial conducted from September 2015 until January 2017 (means, standard deviations and $P$ values adjusted for age and sex)

\begin{tabular}{|c|c|c|c|c|c|c|c|c|c|c|}
\hline & \multicolumn{5}{|c|}{ Anaemia† } & \multicolumn{5}{|c|}{ HIV } \\
\hline & \multicolumn{2}{|c|}{ Anaemic (n91) } & \multicolumn{2}{|c|}{$\begin{array}{c}\text { Non-anaemic } \\
(n 77)\end{array}$} & \multirow[b]{2}{*}{$P$ value } & \multicolumn{2}{|c|}{$\begin{array}{l}\text { HIV positive } \\
\text { (n 35) }\end{array}$} & \multicolumn{2}{|c|}{$\begin{array}{l}\text { HIV negative } \\
\quad(n 31)\end{array}$} & \multirow[b]{2}{*}{$P$ value } \\
\hline & Mean & SD & Mean & SD & & Mean & SD & Mean & SD & \\
\hline Erythrocytes $\left(\times 10^{12} /\right)$ & 4.6 & 0.1 & 4.7 & 0.1 & 0.229 & $3 \cdot 7$ & 0.2 & 4.9 & 0.2 & $<0.001$ \\
\hline Platelets $\left(\times 10^{9} /\right)$ & 434 & 17 & 371 & 18 & 0.010 & 349 & 34 & 343 & $4 \cdot \overline{3}$ & 0.915 \\
\hline Leucocytes $\left(\times 10^{9} / \mathrm{I}\right)$ & $12 \cdot 1$ & 0.5 & 11.4 & 0.5 & 0.306 & 8.3 & 0.8 & $12 \cdot 2$ & 0.8 & 0.005 \\
\hline Neutrophils $\left(\times 10^{9} / I\right)$ & 0.6 & 0.0 & 0.6 & 0.0 & 0.950 & $2 \cdot 8$ & 0.5 & 4.9 & 0.5 & 0.006 \\
\hline Lymphocytes $\left(\times 10^{9} / l\right)$ & $6 \cdot 4$ & 0.3 & $5 \cdot 8$ & 0.3 & 0.237 & $4 \cdot 7$ & 0.5 & $6 \cdot 0$ & 0.5 & 0.125 \\
\hline Monocytes $\left(\times 10^{9} / /\right)$ & 0.9 & 0.1 & 0.8 & 0.1 & 0.176 & 0.7 & $0 \cdot 1$ & 0.8 & 0.1 & 0.250 \\
\hline \multirow[t]{4}{*}{ Eosinophils $\left(\times 10^{9} / \mathrm{l}\right)$} & 0.3 & 0.0 & 0.3 & 0.0 & 0.740 & 0.2 & $0 \cdot 1$ & 0.4 & $0 \cdot 1$ & 0.167 \\
\hline & \multicolumn{5}{|c|}{ Inflammation§ } & \multicolumn{5}{|c|}{ Haemoglobinopathies } \\
\hline & \multicolumn{2}{|c|}{$\begin{array}{c}\text { Inflammation } \\
(n 65)\end{array}$} & \multicolumn{2}{|c|}{$\begin{array}{c}\text { No inflammation } \\
(n 96)\end{array}$} & \multirow[b]{2}{*}{$P$ value } & \multicolumn{2}{|c|}{$\begin{array}{l}\text { Hb disorder } \\
(n 36)\end{array}$} & \multicolumn{2}{|c|}{$\begin{array}{l}\text { No Hb disorder } \\
(n 8)\end{array}$} & \\
\hline & Mean & SD & Mean & SD & & Mean & SD & Mean & SD & $P$ value \\
\hline Erythrocytes $\left(\times 10^{12} / l\right)$ & 4.5 & 0.1 & 4.7 & $0 \cdot 1$ & 0.069 & 4.8 & 0.1 & 4.8 & 0.1 & 0.795 \\
\hline Platelets $\left(\times 10^{9} / \mathrm{l}\right)$ & 462 & 20 & 386 & 16 & 0.002 & 481 & 27 & 436 & 20 & 0.179 \\
\hline Leucocytes $\left(\times 10^{9} / \mathrm{I}\right)$ & $12 \cdot 6$ & 0.5 & 11.5 & 0.4 & 0.073 & 1.2 & 0.7 & 12.9 & 0.5 & 0.140 \\
\hline Neutrophils $\left(\times 10^{9} / I\right)$ & $5 \cdot 2$ & 0.3 & $5 \cdot 1$ & 0.2 & 0.001 & 0.7 & 0.0 & 0.6 & 0.0 & 0.179 \\
\hline Lymphocytes $\left(\times 10^{9} / l\right)$ & $6 \cdot 1$ & 0.4 & $6 \cdot 2$ & 0.3 & 0.836 & $7 \cdot 4$ & 0.4 & $7 \cdot 2$ & 0.4 & 0.637 \\
\hline Monocytes $\left(\times 10^{9} / /\right)$ & 0.9 & 0.1 & 0.8 & 0.5 & 0.052 & 1.0 & 0.1 & 1.0 & 0.1 & 0.635 \\
\hline Eosinophils $\left(\times 10^{9} / 1\right)$ & 0.3 & 0.1 & 0.3 & 0.1 & 0.583 & 0.5 & 0.1 & 0.3 & 0.1 & 0.021 \\
\hline
\end{tabular}

†Analyses were not adjusted for age and sex, as this is included in the diagnostic criteria. ¥Only including children who had been tested for HIV.

§Inflammation was defined as C-reactive protein $>5 \mathrm{mg} / \mathrm{l}$ or $\alpha_{1}$-acid glycoprotein $>1 \mathrm{~g} / \mathrm{l}$.

(Table 3), and high monocyte count was associated with high total PUFA, AA, $n-6$ LCPUFA, DHA and $n$ - 3 LCPUFA (Tables 3 and 4).

\section{Comparing blood cell counts in children with and without health challenges}

There was no significant difference observed in blood cell counts between children with SAM and MAM (see online supplementary material, Supplemental Table S3). Children with anaemia appeared to have a higher mean platelet count than those without anaemia $\left(\times 10^{9} / 1\right.$ : $434 v$. 371, $P=0.010$; Table 5). Furthermore, an association analysis adjusted for age and sex showed a non-significant tendency towards a negative correlation between $\mathrm{Hb}$ concentration and platelet count $\left(\times 10^{9} / 1:-16 \cdot 8 ; 95 \% \mathrm{CI}-33 \cdot 8\right.$, $0 \cdot 2)$, but not for monocyte count $\left(\times 10^{9} / 1\right.$ : -0.00 ; $95 \%$ CI $-0.05,0.05)$. Presence of inflammation $v$. no inflammation resulted in a significantly higher mean platelet count $\left(\times 10^{9} / 1\right.$ : $\left.462 v .386, P=0.002\right)$ and a strong tendency for a higher mean monocyte count $\left(\times 10^{9} / 1\right.$ : 0.9 v. 0.8 , $P=0.052$; Table 5). Children with inflammation defined as $\mathrm{CRP}>5 \mathrm{mg} / 1$ ( 72 counts; $95 \%$ CI 9, 135 counts) or AGP $>1 \mathrm{~g} / 1$ (74 counts; $95 \%$ CI 25, 123 counts) had higher mean platelets $\left(\times 10^{9} / 1\right)$ compared with children with no signs of infection. Monocyte counts were elevated in children with CRP $>5 \mathrm{mg} / 1$ (estimated difference, $\times 10^{9} / 1$ : 0.20 counts; $95 \%$ CI $0.01,0.39$ counts) compared with children with normal CRP, but not in children with AGP above the cut-off of $>1 \mathrm{~g} / 1$ (estimated difference, $\times 10^{9} / 1$ : 0.09 counts; $95 \% \mathrm{CI}-0 \cdot 06,0.25$ counts). Hence, the association was dependent on the stage of inflammation.

Children with HIV had lower counts of erythrocytes, leucocytes and neutrophils (Table 5). The presence of haemoglobinopathies was significantly associated with a higher mean eosinophil count $\left(\times 10^{9} / 1: 0.5 v\right.$. 0.3 , $P=0.021)$ and a tendency towards a lower mean platelet count $\left(\times 10^{9} / 1\right.$ : $481 v$. 436, $\left.P=0 \cdot 179\right)$.

\section{Discussion}

We found low relative contributions of total whole-blood PUFA in children admitted to outpatient treatment of acute malnutrition. Stunting and low height were associated with low total PUFA, LA, AA and n-6 LCPUFA. Low mid-upperarm muscle area and high mid-upper-arm fat percentage were also associated with low AA and n-6 LCPUFA, but no associations were observed for wasting or any other measures of weight except for EPA and total weight. No associations were observed between $\mathrm{Hb}$, CRP or AGP, and total PUFA, sub-classes of PUFA or any of the individual PUFA. The presence of HIV infection, progressed inflammation (defined by AGP) and haemoglobin- 
opathies were associated with high MA:AA, and hence a risk of very low $n-6$ PUFA status. The presence of haemoglobinopathies was also associated with low total PUFA, especially in the young children. High platelet and monocyte counts appeared to be linked with inflammation and anaemia. High platelet and monocyte counts were also associated with high total PUFA and LA and with $n-6$ and $n-3$ LCPUFA, respectively.

\section{Whole-blood PUFA levels}

The acutely malnourished children in the current study had considerably lower total PUFA, $n-6$ and $n-3$ PUFA, and AA and DHA compared with a population of rural Cambodian infants $^{(49)}$ living in an environment with limited dietary resources $^{(51)}$ and vulnerable to stunting and wasting ${ }^{(52)}$, respectively. The whole-blood ALA assessed in the present study children diagnosed with acute malnutrition was similar to what was reported in children with MAM from Burkina Faso $(0.2 \% \mathrm{FA})^{(16)}$, while a study in Ugandan children with a more severe stage of SAM found even lower ALA (0.15\% FA) as well as lower LA (10.5 v. $13.2 \% \mathrm{FA}$ in the present study) ${ }^{(18)}$. We found relatively low MA (0.08\% FA) compared with well-nourished Ugandan children ( $0 \cdot 10 \% \mathrm{FA})$, which was unexpected, since the Ugandan children with SAM had higher whole-blood MA $(0.12 \% \mathrm{FA})^{(18)}$. Our results are in line with the MAM study in Burkina Faso with regard to the essential fatty acid deficiency markers of MA:AA, n-6 DPA:DHA and $n-6: n-3$ PUFA $^{(16)}$.

Low PUFA in acutely malnourished children could be due to infection and metabolic disturbances associated with malnutrition ${ }^{(15)}$. These disturbances could directly impair lipid digestion and uptake ${ }^{(15)}$ and also indirectly be affected by other nutritional imbalances such as Fe deficiency, which, in combination with acute malnutrition, could impact on PUFA metabolism through reduced $\Delta$-6desaturase enzyme activity ${ }^{(53)}$ and consequently reduced capacity for the conversion of LA and ALA to their longchain metabolites.

Whole-blood PUFA is highly influenced by diet, rather than by malnutrition per $s e^{(10)}$. Cambodian children generally consume a diet based on rice, with limited amounts of vegetables, legumes and fruits, and little or no animalsource foods ${ }^{(3,4)}$. This diet is at risk of being low in PUFA. Also, the cooking oils typically used are low in $n-3$ fatty acids ${ }^{(4)}$. Fish and fish products are the main animal-source food in the typical Cambodian $\operatorname{diet}^{(54)}$ and, overall, fish is a highly important and valuable PUFA source in diets. The fish in the Cambodian diet are primarily freshwater fish available from the rich aquatic environment of the Mekong river basin. Tropical freshwater fish are generally lower in DHA and EPA compared with cold-water marine fish species ${ }^{(55-57)}$. Furthermore, despite fish being consumed very frequently in Cambodia (even daily in the peak fishing season) the portion size can be very small, especially for children, resulting in low overall fish intake ${ }^{(51)}$ and a diet low in $n$-3 PUFA. The low PUFA status of the children in our study could therefore also be the result of a low dietary intake, further enforced by the suffering from acute malnutrition.

\section{Nutritional markers associated with whole-blood $\boldsymbol{P U F A}$}

The associations between stunting, height and low n-6 PUFA support the idea that sufficient dietary PUFA intake may be important in preventing stunting. Height gain was found to be correlated with higher DHA in young children in treatment for SAM in Uganda(17). A study of 2-6-year-old children from Tanzania found associations between risk of stunting and low wholeblood LA and total $n-6$ PUFA $^{(58)}$, while mixed findings between stunting and PUFA were reported in Ghanaian children ${ }^{(59,60)}$. The indication of a biological link between linear growth and PUFA status could be mediated by eicosanoids such as AA-derived prostaglandin $\mathrm{E}_{2}$, which can affect proteolysis in skeletal muscle and thereby have an impact on linear growth ${ }^{(61)}$. Another pathway could go through prostaglandin $\mathrm{E}_{2}$ stimulation of insulin-like growth factor-1 expression, which again meditates bone Ca accretion ${ }^{(10,58,62)}$. A few studies have found increased insulin-like growth factor-1 after fish-oil supplementation in children ${ }^{(63,64)}$.

We observed an association between high PUFA and muscle mass. Low mid-upper-arm fat percentage and high mid-upper-arm muscle area were associated with high AA and n-6 LCPUFA, meaning that high fat percentage or a reduced muscle mass was associated with less PUFA in the blood. Acute malnutrition causes muscle atrophy, which could be linked to increased stress metabolism and hypercortisolaemia $^{(65)}$. Although beyond the conclusions of the present study, we speculate that hypercortisolaemia may promote muscle proteolysis and increase energy expenditure and fat oxidation ${ }^{(66)}$, which could result in a combined effect of lower PUFA and muscle mass. Cortisol and muscle proteolysis as well as low PUFA could also be caused by the combination of low dietary intake and malnutrition.

\section{Compromised bealth is associated with very low PUFA status}

$\mathrm{Hb}$ concentration and anaemia were not associated with any PUFA or the cut-off used to define very low PUFA status. This is inconsistent with studies of children with either MAM or SAM that show a correlation between Hb concentration and AA and DHA and low PUFA status (n-6 DPA: DHA and MA:AA) ${ }^{(16,18)}$.

Other studies observed an association between acute inflammation (CRP $>5 \mathrm{mg} / \mathrm{l})$ and PUFA (LA, EPA and $n-3$ DPA) in children with $\mathrm{SAM}^{(18)}$ and between progressed inflammation (AGP $\geq 1 \mathrm{~g} / \mathrm{l}$ ) and PUFA (AA, $n-6$ DPA, ALA 
and DHA) in children with MAM ${ }^{(16)}$. The present study's finding that the inflammation markers were not associated with PUFA could probably be because the prevalence of inflammation was low compared with the two other studies $^{(16,18)}$. Studies in various populations have shown reduced CRP following fish-oil supplementation ${ }^{(67-69)}$, which could support the suggestion that high PUFA may have a protective effect against inflammation.

Haemoglobinopathies were associated with low total PUFA in an age-stratified analysis in the young children. We speculate that haemoglobinopathies could cause a higher erythrocyte turnover, and thereby a higher phospholipid turnover. In the presence of haemoglobinopathies, a higher repair of blood cells, required due to oxidant stress and lipid damage of the erythrocyte membrane, is also speculated. Furthermore, because of lipid oxidation, the phospholipid repair system is insufficient in maintaining a proper molecular fatty acid composition in erythrocytes.

All blood cells are exposed to the same pool of fatty acid substrate from plasma, and the difference in PUFA measured in the whole-blood samples could be caused by the activity of enzymes using the fatty acid pool for phospholipid repair. Oxidant stress in erythrocytes may challenge the incorporation of PUFA, which affects the fatty acid composition of the membrane ${ }^{(70)}$. This may lead to low PUFA in cells and thereby to lower PUFA in children with haemoglobinopathies than in children without haemoglobinopathies.

\section{Health markers' influence on blood counts may indirectly implicate incorrect assessment of whole-blood PUFA status}

Unexpectedly, the inflammation markers were not associated with any PUFA but were found to be associated with counts of platelets and monocytes, also formed as a response to infection. A sub-analysis showed an association between the markers of inflammation and platelets and monocytes, which confirms the expected biological response of CRP and AGP at the onset of infection and inflammation. The pattern of associations between the different markers used to define inflammation indicated differences in the biological response to acute and progressed inflammation.

Different blood fractions and cell types contribute different PUFA levels, so changes in the number of a specific cell could shift levels of PUFA in whole blood. This is likely to explain the increased PUFA we observed with increased monocyte and platelet counts, as monocytes consist of $\sim 20 \%$ AA and AA increases with increased monocyte count $^{(71)}$. Also, a decrease in erythrocytes, which is likely to be associated with anaemia, could result in reduced measurement of PUFA in whole-blood samples. These contra-acting mechanisms could explain the lack of associations of health markers for anaemia, inflammation and haemoglobinopathies with PUFA in the present study.
The combined effects of anaemia with elevated platelets and monocytes caused by inflammation may have masked changes in PUFA.

The fatty acid composition of immune cells (e.g. lymphocytes and monocytes) is also expected to alter in accordance with dietary intake, which impacts the capacity of the immune cells to produce eicosanoids, which are involved in immune system regulation ${ }^{(72)}$. Thus, there is a two-way association between the immune system and PUFA. Inflammation is suggested to cause elevated cell counts in the present study, which may lead to misinterpretation of PUFA in whole blood. Inflammation markers were observed to influence platelets and monocytes differently, and platelets and monocytes were not associated with the same fatty acids. The complexity of these associations would make it difficult to adjust for inflammation markers and cell counts when assessing PUFA in whole blood in populations with severe health challenges such as acute malnutrition.

\section{Limitations}

The present study shares the limitation of other observational studies in that it identifies associations but does not identify causality. Another limitation is that we did not include data on total food consumption covering all dietary sources of PUFA such as quantity of fish and breast milk consumed. Also, the predefined cut-offs used to estimate essential fatty acid deficiencies were derived from infants and were applied to older children in the present study. The stage of deficiencies in children above infancy could be different. Finally, all the health markers used in the present study are to some degree related through cell counts, and it could be argued that all health markers should be adjusted for each other (anaemia, inflammation, HIV infection and haemoglobinopathies). Our study used secondary data and unfortunately it did not have insufficient power to adjust all health challenges for each of the others.

\section{Conclusion}

Overall, children with acute malnutrition in Cambodia had low total whole-blood PUFA and indicated to have a high prevalence of a very low PUFA. HAZ (stunting) and absolute height were both positively associated with PUFA, whereas no associations were found for wasting and other weight measures. Elevated platelets and monocytes, likely due to inflammation, were positively associated with PUFA, thus indicating that blood cell counts influence wholeblood PUFA levels. Therefore, in acutely malnourished Cambodian children, inflammation that elevates blood cell counts could result in increased blood levels of PUFA that do not reflect dietary intake and nutritional status. These findings indicate the need for further studies on the relationship between stunting and PUFA in acutely 
malnourished children through randomized controlled trials with dietary PUFA supplementation. Furthermore, future field studies using whole blood and dried blood spots to assess PUFA status should consider co-morbidities associated with severe health challenges, such as malnourishment, that can change the cellular composition of blood.

\section{Acknowledgements}

Acknowledgements: The authors would like to thank the National Pediatric Hospital for its collaboration and support in conducting the study. Deep thanks are extended to all caregivers, the participating children and the data collection team. Financial support: The study was financially supported by UNICEF's national committees (Australia, Republic of Korea, Hong Kong), Institute de Recherché pour le Développment, University of Copenhagen and Neys-van Hoogstraten Foundation (grant number CA271). The funding bodies had no influence on data collection, data analysis or publication of the results. Conflict of interest: The authors have no conflicts of interest to declare. The views expressed are those of the authors and do not reflect the official policies or opinions of the organizations to which they belong. Authorship: S.S., F.T.W., A.L., C.C. and N.R. designed the study and formulated the research questions; S.S., N.A. and D.M. collected the data; S.S drafted the initial manuscript and analysed the data; L.L., F.T.W., K.D.S. and N.R. contributed to the interpretation of the data and the manuscripts; all authors reviewed and approved the final manuscript. Ethics of human subject participation: This study was conducted according to the guidelines laid down in the Declaration of Helsinki and all procedures involving research study participants were approved by the National Ethical Committee for Health Research of the Ministry of Health, Kingdom of Cambodia (April 2015 Version No. 2). Both written and verbal informed consent were obtained from all participants. The trial was registered at ClinicalTrials.gov (name: Comparison of a Locally Produced RUTF With a Commercial RUTF in the Treatment of SAM (FLNS_SAM); trial registration: NCT02907424; URL: https://clinicaltrials.gov/ct2/show/NCT02907424).

\section{Supplementary material}

To view supplementary material for this article, please visit https://doi.org/10.1017/S1368980019003744

\section{References}

1. Black RE, Victora CG, Walker SP et al. (2013) Maternal and child undernutrition and overweight in low-income and middle-income countries. Lancet 382, 427-451.

2. Lapidus N, Luquero FJ, Gaboulaud V et al. (2009) Prognostic accuracy of WHO growth standards to predict mortality in a large-scale nutritional program in Niger. PLoS Med 6, 0278-0285.

3. Fisheries Administration, Inland Fisheries Research and Development Institute (2012) Food and Nutrition Security Vulnerability to Mainstream Hydropower Dam Development in Cambodia. Phnom Penh: Fisheries Administration.

4. Michaelsen KF, Hoppe CA, Roos N et al. (2009) Choice of foods and ingredients for moderately malnourished children 6 months to 5 years of age. Food Nutr Bull 30, 3 Suppl., S343-S404.

5. Lauritzen L, Hansen HS, Jørgensen MH et al. (2001) The essentiality of long chain $n-3$ fatty acids in relation to development and function of the brain and retina. Prog Lipid Res 40, $1-94$

6. Jacobsen JL, Jacobson SW, Muckle G et al. (2008) Beneficial effects of a polyunsaturated fatty acid on infant development. J Pediatr 152, 356-364.

7. Ratnayake WMN \& Galli C (2009) Fat and fatty acid terminology, methods of analysis and fat digestion and metabolism: a background review paper. Ann Nutr Metab 55, 8-43.

8. Holman RT, Johnson SB, Osvaldo M et al. (1981) Essential fatty acid deficiency in malnourished children. Am J Clin Nutr 34, 1534-1539.

9. Crane RJ, Jones KDJ \& Berkley JA (2015) Environmental enteric dysfunction: an overview. Food Nutr Bull 36, 76-87.

10. Smit EN, Muskiet FAJ \& Boersma ER (2004) The possible role of essential fatty acids in the pathophysiology of malnutrition: a review. Prostaglandins Leukot Essent Fatty Acids 71, 241-250.

11. Koletzko B, Abiodun PO, Laryea MD et al. (1986) Fatty acid composition of plasma lipids in Nigerian children with protein-energy malnutrition. Eur J Pediatr 145, 109-115.

12. Taylor GO (1971) Serum triglycerides fatty acids nonesterified in kwashiorkor. Am J Clin Nutr 24, 1212-1215.

13. Naismith $D$ (1973) Kwashiorkor in western Nigeria: a study of traditional weaning foods, with particular reference to energy and linoleic acid. Br J Nutr 30, 567-576.

14. Franco VH, Hotta JK, Jorge SM et al. (1999) Plasma fatty acids in children with grade III protein-energy malnutrition in its different clinical forms: marasmus, marasmic kwashiorkor, and kwashiorkor. J Trop Pediatr 45, 71-55.

15. Smit EN, Dijkstra JM, Schnater TA et al. (1997) Effects of malnutrition on the erythrocyte fatty acid composition and plasma vitamin E levels of Pakistani children. Acta Paediatr 86, 690-695.

16. Yaméogo CW, Cichon B, Fabiansen C et al. (2017) Correlates of whole-blood polyunsaturated fatty acids among young children with moderate acute malnutrition. Nutr J 16, 44-55.

17. Babirekere-Iriso E, Mortensen CG, Mupere E et al. (2016) Changes in whole-blood PUFA and their predictors during recovery from severe acute malnutrition. Br J Nutr 115, 1730-1739.

18. Babirekere-Iriso E, Lauritzen L, Mortensen CG et al. (2016) Essential fatty acid composition and correlates in children with severe acute malnutrition. Clin Nutr ESPEN 11, 40-46.

19. Brenna JT, Plourde M, Stark KD et al. (2018) Best practices for the design, laboratory analysis, and reporting of trials involving fatty acids. Am J Clin Nutr 108, 211-227.

20. Bachou H, Tylleskär T, Kaddu-Mulindwa DH et al. (2006) Bacteraemia among severely malnourished children infected and uninfected with the human immunodeficiency virus-1 in Kampala, Uganda. BMC Infect Dis 6, 160.

21. Babirekere-Iriso E, Musoke P \& Kekitiinwa A (2006) Bacteraemia in severely malnourished children in an HIVendemic setting. Ann Trop Paediatr 26, 319-328.

22. Jones KDJ \& Berkley JA (2014) Severe acute malnutrition and infection. Paediatr Int Child Health 34, Suppl. 1, S1-S29.

23. Jones KD \& Berkley JA (2014) Childhood malnutrition: toward an understanding of infections, inflammation, and antimicrobials. Food Nutr Bull 35, 64-70. 
24. Thakur N, Chandra J, Pemde H et al. (2014) Anemia in severe acute malnutrition. Nutrition 30, 440-442.

25. Ingersoll MA, Platt AM, Potteaux S et al. (2011) Monocyte trafficking in acute and chronic inflammation. Trends Immunol 32, 470-477.

26. Yoshida SH, Keen CL, Ansari AA et al. (1999) Nutrition and the immune system. In Modern Nutrition in Health and Disease, 9th ed., pp. 725-750 [ME Shils, JA Olson, M Shike et al., editors]. Philadelphia, PA: Lippincott Williams \& Wilkins.

27. Weatherall DJ \& Clegg JB (2001) Inherited haemoglobin disorders: an increasing global health problem. Bull World Health Organ 79, 704-712.

28. Fucharoen S \& Weatherall DJ (2012) The hemoglobin E thalassemias. Cold Spring Harb Perspect Med 2, a011734.

29. Sigh S, Roos N, Chamnan C et al. (2018) Effectiveness of a locally produced, fish-based food product on weight gain among Cambodian children in the treatment of acute malnutrition: a randomized controlled trial. Nutrients 10, 909-925.

30. Sigh S, Roos N, Sok D et al. (2018) Development and acceptability of fish-based, ready-to-use products for the prevention and treatment of malnutrition in Cambodia. Food Nutr Bull 30, 420-434.

31. World Health Organization \& UNICEF (2009) WHO Child Growth Standards and the Identification of Severe Acute Malnutrition in Infants and Children: A Joint Statement. Geneva: WHO.

32. de Onis M, Onyango AW, Borghi E et al. (2007) Development of a WHO growth reference for school-aged children and adolescents. Bull World Health Organ 85, 812-819.

33. Rolland-Cachera MF, Brambilla P, Manzoni P et al. (1997) Body composition assessed on the basis of arm circumference and triceps skinfold thickness: a new index validated in children by magnetic resonance imaging. Am J Clin Nutr 65, 1709-1713.

34. Metherel AH, Aristizabal Henao JJ \& Stark KD (2013) EPA and DHA levels in whole blood decrease more rapidly when stored at $-20^{\circ} \mathrm{C}$ as compared with room temperature, 4 and $-75^{\circ} \mathrm{C}$. Lipids $\mathbf{4 8}, 1079-1091$.

35. Metherel AH \& Stark KD (2015) Cryopreservation prevents iron-initiated highly unsaturated fatty acid loss during storage of human blood on chromatography paper at -20 degrees C. J Nutr 145, 654-660.

36. Marangoni F, Colombo C \& Galli C (2004) A method for the direct evaluation of the fatty acid status in a drop of blood from a fingertip in humans: applicability to nutritional and epidemiological studies. Anal Biochem 326, 267-272.

37. Metherel AH, Taha AY, Izadi H et al. (2009) The application of ultrasound energy to increase lipid extraction throughput of solid matrix samples (flaxseed). Prostaglandins Leukot Essent Fatty Acids 81, 417-423.

38. Metherel AH, Buzikievich LM, Charkhzarin P et al. (2012) Omega-3 polyunsaturated fatty acid profiling using fingertip-prick whole blood does not require overnight fasting before blood collection. Nutr Res 32, 547-556.

39. Armstrong JM, Metherel AH \& Stark KD (2008) Direct microwave transesterification of fingertip prick blood samples for fatty acid determinations. Lipids $\mathbf{4 3}, 187-196$.

40. Lauritzen L, Sørensen LB, Harsløf LB et al. (2017) Mendelian randomization shows sex-specific associations between long-chain PUFA-related genotypes and cognitive performance in Danish schoolchildren. Am J Clin Nutr 106, 88-95.

41. Holman RT (1971) Essential fatty acid deficiency. In Polyunsaturated Fatty Acids, pp. 275-348 [RT Holman, editor]. New York: Pergamon Press.

42. Bourre JM, Francois M, Youyou A et al. (1989) The effects of dietary alpha-linolenic acid on the composition of nerve membranes, enzymatic activity, amplitude of electrophysiological parameters, resistance to poisons and performance of learning tasks in rats. J Nutr 119, 1880-1892.
43. World Health Organization (2011) Haemoglobin Concentrations for the Diagnosis of Anaemia and Assessment of Severity. Geneva: WHO.

44. Wieringa FT, Dahl M, Chamnan C et al. (2016) The high prevalence of anemia in Cambodian children and women cannot be satisfactorily explained by nutritional deficiencies or hemoglobin disorders. Nutrients 8, 348-360.

45. Erhardt JG, Estes JE, Pfeiffer CM et al. (2004) Combined measurement of ferritin, soluble transferrin receptor, retinol binding protein, and C-reactive protein by an inexpensive, sensitive, and simple sandwich enzyme-linked immunosorbent assay technique. J Nutr 134, 3127-3132.

46. Thurnham DI, McCabe LD, Haldar S et al. (2010) Adjusting plasma ferritin concentrations to remove the effects of subclinical inflammation in the assessment of iron deficiency: a meta-analysis. Am J Clin Nutr $92,546-555$.

47. Dalmeijer GW, Wijga AH, Gehring U et al. (2016) Fatty acid composition in breastfeeding and school performance in children aged 12 years. Eur J Nutr 55, 2199-2207.

48. Decsi T, Thiel I \& Koletzko B (1995) Essential fatty acids in full-term infants fed breast milk or formula. Arch Dis Child Fetal Neonatal Ed 72, 23-28.

49. Nurhasan M, Roos N, Skau JK et al. (2018) Effect of complementary food with small amounts of freshwater fish on whole blood $n-3$ fatty acids in Cambodian infants age 6-15 months. Prostaglandins Leukot Essent Fatty Acids 135, 92-101.

50. Barbarich BN, Willows ND, Wang L et al. (2006) Polyunsaturated fatty acids and anthropometric indices of children in rural China. Eur J Clin Nutr 60, 1100-1107.

51. Skau JKH, Bunthang T, Chamnan C et al. (2014) The use of linear programming to determine whether a formulated complementary food product can ensure adequate nutrients for 6- to 11-month-old Cambodian infants. Am J Clin Nutr 99 , $130-138$

52. Skau JKH, Touch B, Chhoun C et al. (2015) Effects of animal source food and micronutrient fortification in complementary food products on body composition, iron status, and linear growth: a randomized trial in Cambodia. Am J Clin Nutr 101, 742-751.

53. Smuts CM, Tichelaar HY, Jaarsveld PJ Van et al. (1995) The effect of iron fortification on the fatty acids composition of plasma and erythrocyte membranes in primary school children with and without iron deficiency. Prostaglandins Leukot Essent Fatty Acids 52, 59-67.

54. Fisheries Administration (2012) Baseline Assessment of Diet and Nutrition in Cambodia 2011. Phnom Penh: Fisheries Administration.

55. Karapanagiotidis IT, Yakupitiyage A, Little DC et al. (2010) The nutritional value of lipids in various tropical aquatic animals from rice-fish farming systems in northeast Thailand. J Food Compost Anal 23, 1-8.

56. Nurhasan M, Maehre HK, Malde MK et al. (2010) Nutritional composition of aquatic species in Laotian rice field ecosystems. J Food Compost Anal 23, 205-213.

57. Li G, Sinclair AJ \& Li D (2011) Comparison of lipid content and fatty acid composition in the edible meat of wild and cultured freshwater and marine fish and shrimps from China. J Agric Food Chem 59, 1871-1881.

58. Jumbe T, Comstock SS, Hahn SL et al. (2016) Whole blood levels of the $n-6$ essential fatty acid linoleic acid are inversely associated with stunting in 2-to-6 year old Tanzanian children: a cross-sectional study. PLoS One 11, $\mathrm{e} 0154715$.

59. Adjepong M, Yakah W, Harris W et al. (2018) Association of whole blood fatty acids and growth in southern Ghanaian children 2-6 years of age. Nutrients 10, 954-968.

60. Adjepong M, Pickens CA, Jain R et al. (2018) Association of whole blood $n-6$ fatty acids with stunting in 2-to-6-year-old 
northern Ghanaian children: a cross-sectional study. PLOS One 13, e0193301.

61. Rodemanns HP \& Goldberg AL (1982) Arachidonic acid, prostaglandin $\mathrm{E}_{2}$ and $\mathrm{F}_{2 \alpha}$ influence rates protein turnover in skeletal and cardiac muscle. J Biol Chem 257, 1632-1638.

62. Harada SI, Balena R, Rodan GA et al. (1995) The role of prostaglandins in bone formation. Connect Tissue Res $\mathbf{3 1}$, 279-282.

63. Damsgaard CT, Harslof LBS, Andersen AD et al. (2016) Fish oil supplementation from 9 to 18 months of age affects the insulin-like growth factor axis in a sex-specific manner in Danish infants. Br J Nutr 115, 782-790.

64. Damsgaard CT, Mølgaard C, Matthiessen J et al. (2012) The effects of $n$-3 long-chain polyunsaturated fatty acids on bone formation and growth factors in adolescent boys. Pediatr Res 71, 713-719.

65. Manary MJ, Muglia LJ, Vogt SK et al. (2006) Cortisol and its action on the glucocorticoid receptor in malnutrition and acute infection. Metab Clin Exp 55, 550-554.

66. Brillion DJ, Zheng BM, Campbell RG et al. (1994) Effect of cortisol on energy expenditure and amino acid metabolism in humans. Am Physiol Soc 268, 501-513.
67. Bowden RG, Wilson RL, Deike E et al. (2009) Fish oil supplementation lowers C-reactive protein levels independent of triglyceride reduction in patients with end-stage renal disease. Nutr Clin Pract 24, 508-512.

68. Saifullah A, Watkins BA, Saha C et al. (2007) Oral fish oil supplementation raises blood omega-3 levels and lowers C-reactive protein in haemodialysis patients - a pilot study. Nephrol Dial Transplant 22, 3561-3567.

69. Ciubotaru I, Lee YS \& Wander RC (2003) Dietary fish oil decreases C-reactive protein, interleukin-6, and triacylglycerol to HDL-cholesterol ratio in postmenopausal women on HRT. J Nutr Biochem 14, 513-521.

70. Kuypers SFA, Cappellini MD, Vichinsky E et al. (2007) RBCs and hemoglobinopathies membrane lipid alterations in hemoglobinopathies. Hematology Am Soc Hematol Educ Program issue $1,68-73$.

71. Calder PC (2008) The relationship between the fatty acid composition of immune cells and their function. Prostaglandins Leukot Essent Fatty Acids 79, 101-108.

72. Calder PC, Yaqoob P, Thies F et al. (2002) Fatty acids and lymphocyte functions. Br J Nutr 87, Suppl. 1, S31-S48. 